\title{
5. Katalog der Spolien
}

\author{
Abkürzungen: \\ LM: Theodosianische Landmauer \\ BM: Blachernen-Mauer \\ SM: Marmara-Seemauer \\ GHM: Goldene Horn-Mauer \\ TS: Tekfur Saray1 \\ T: Turm \\ K: Kurtine \\ Türme und Kurtinen mit dem Buchstaben a beziehen sich wieder auf die Vormauer. \\ Datierungsvorschläge stammen überwiegend von der Verfasserin
}

\subsection{Kapitelle}

K-1. Korinthisches Säulenkapitell

Marmor

Ort: LM, T. 2, Innenraum

Maße: Höhe 80 , obere Breite $82 \mathrm{~cm}$

Beschreibung: Zwei Blattkränze, Eckvoluten, zweistreifiges Abakusprofil, gekerbte Abakusbosse. Großgezahnte, fünflappige Akanthusblätter des Kranzes sowie der Hochblätter schließen sich durch Spitzenberührung zusammen. Die mittleren Rippen der Hochblätter mit überfallenden Blattspitzen. Der mittlere Teil des linken Volutenstengels, die überfallenden Blätter und der Kapitellfuß sind beschädigt.

Datierung: 5. Jahrhundert

Literatur: Ahunbay, Land Walls, 231, Abb. 6.

K-2. Korinthisches Säulenkapitell

Marmor

Ort: LM, T. 2, Innenraum

Maße: Höhe 80 , obere Breite $82 \mathrm{~cm}$

Beschreibung: Zwei Blattkränze, verwilderte Eckvoluten, zweistreifiges Abakusprofil, gekerbte Abakusbosse. Großgezahnte, fünflappige Akanthusblätter des Kranzes sowie der Hochblätter schließen sich durch Spitzenberührung zusammen. Die wulstigen mittleren Rippen mit überfallenden Blattspitzen. Unter der Abakusbosse (Kernmassenbuckel) Steinmetzzeichen $\Xi+\Theta$. Volutenecken, Kapitellfuß und der untere Teil des Blattkranzes beschädigt.

Datierung: 5. Jahrhundert

Literatur: Wie K-1

K-3. Dreizoniges Kompositkapitell

Marmor

Ort: LM, Vorwerk des Goldenen Tors, Feldseite, Süden

Maße konnten nicht aufgenommen werden 
Beschreibung: Halsring und Fußwulst Perlstab, eine Reihe überfallender, feingezahnter Blättchen. Hoher Blattkranz aus acht eng anliegenden, feingezahnten Akanthusblättern mit breit überfallenden Blattspitzen, durch Reihen von Bohrlöchern akzentuiert. Darüber Kragen mit Perlstab und Kranz aus weit überstehenden Palmetten. Auf dem oberen Kalathosrand Kragen aus aufrecht stehenden Palmetten. Auf dem mittleren Blattkranz sitzende Tauben unter den weit vorspringenden Abakusecken. Die Mittelbosse in Gestalt einer Taubenprotome.

Datierung: 5. Jahrhundert

Literatur: Strzygowski, Goldenes Thor, 27, Abb. 17; Zollt, Kapitellplastik, 225, Nr. 649 (Bibl.).

Abb. 124

K-4. Dreizoniges Kompositkapitell

Marmor

Ort: LM, Vorwerk des Goldenen Tors, Feldseite, Norden

Wie K-3

Abb. 124

K-5. Fragment eines ionischen Kämpferkapitells

Marmor

Ort: LM, T. 2, Innenraum

Maße: Kapitellteil Höhe 11, Breite $33 \mathrm{~cm}$; Kämpferteil Höhe 21, Breite $68 \mathrm{~cm}$

Beschreibung: Eine Nebenseite sichtbar. Zylindrisches Polster mit zwei vertikalen Linien in der Mitte, von zwei V-förmigen Blättern mit Mittelrippe flankiert. Kämpfer und obere Randleiste schmucklos.

Datierung: 6. Jahrhundert

Unpubliziert

K-6. Fragment eines ionischen Kämpferkapitells

Marmor

Ort: LM, T. 28, Westseite

Maße: Ionischer Teil Höhe 10, Breite $45 \mathrm{~cm}$; Kämpferteil Höhe 20, Breite $50 \mathrm{~cm}$

Beschreibung: Eine Haupt- und eine Nebenseite sichtbar. Hauptseite Kapitellteil: Voluten (die linke beschädigt), Echinus mit großem, herzblattförmig umrahmten Ovulus. Hauptseite Kämpfer: Reliefiertes lateinischen Kreuz mit zwei sich verbreiternden Enden. Nebenseite Kapitellteil: Zylindrisches Polster mit zwei vertikalen Linien in der Mitte, von zwei V-förmigen Blättern flankiert. Nebenseite Kämpfer: Geritztes Kreuz. Datierung: 6. Jahrhundert

Literatur: Meyer-Plath/Schneider, Landmauer, 75.

Abb. 201

K-7. Fragment eines ionischen Kämpferkapitells

Marmor

Ort: LM, T. 28, Westseite

Maße: Wie K-6

Beschreibung: Eine Nebenseite ist sichtbar. Kapitellteil: Zylindrisches Polster mit diagonaler Kreuzkerbung in der Mitte, von Akanthusblättern flankiert. Kämpfer: Geritztes Kreuz, teilweise getilgt.

Datierung: 6. Jahrhundert

Literatur: Wie K-6 
K-8. Fragment eines ionischen Kämpferkapitells

Marmor

Ort: LM, T. 66, Südflanke

Maße: Ionischer Teil Höhe 10, Breite $44 \mathrm{~cm}$; Kämpferteil Höhe 16, Breite $49 \mathrm{~cm}$

Beschreibung: Hauptseite ragt aus dem Mauerverband heraus. Die rechte Volute beschädigt, der Echinus mit einem Ovulus, von stilisierten Blättern flankiert. Breite Trennleiste, Kämpfer schmucklos.

Datierung: 6. Jahrhundert

Unpubliziert

K-9. Fragment eines ionischen Kämpferkapitells

Marmor

Ort: LM, K. 91/92, Stadtseite

Maße: Ionischer Teil Höhe 9, Breite $44 \mathrm{~cm}$; Kämpferteil Höhe 16, Breite $49 \mathrm{~cm}$

Beschreibung: Eine Nebenseite sichtbar. Rechts und links Volutenränder, in der Mitte des zylindrischen Polsters vertikale Leiste, dazwischen gegenständige, gekerbte Blattformen. Kämpfer schmucklos.

Datierung: 6. Jahrhundert

Unpubliziert

K-10. Fragment eines ionischen Kämpferkapitells

Marmor

Ort: SM, K. 17/18, Seeseite

Maße: Höhe 10, Breite $144 \mathrm{~cm}$

Beschreibung: Hauptseite sichtbar. Ionisches Element, flache, wulstige Voluten, in der Mitte des Echinus ein gerahmter Ovulus. Gerade Trennleisten.

Datierung: 6. Jahrhundert

Unpubliziert

K-11. Fragment eines ionischen Kämpferkapitells

Marmor

Ort: SM, K. 17/18, Seeseite

Maße: Höhe 10, Breite $40 \mathrm{~cm}$

Beschreibung: Umgekehrt eingemauert. Hauptseite des ionischen Elements sichtbar. Zwei Voluten, flach ausgebauchter Echinussattel. Ovulus in einer Hülle, beiderseits von dreifingrigen Blättern eingefaßt. Gerade Trennleiste.

Datierung: 6. Jahrhundert

Unpubliziert

K-12. Fragment eines ionischen Kämpferkapitells

Marmor

Ort: SM, K. 19/20, Seeseite

Maße: Höhe 10, Breite $40 \mathrm{~cm}$

Beschreibung: Umgekehrt eingemauert. Hauptseite des ionischen Elements sichtbar. Zwei flache, wulstige Voluten, Echinus mit vorstehendem Ovulus auf einem erhöhten Polster. Trennleiste.

Datierung: 6. Jahrhundert

Unpubliziert

K-13. Fragment eines ionischen Kämpferkapitells

Marmor

Ort: LM, K. 91/92, Stadtseite 
Maße: Höhe $9 \mathrm{~cm}$

Beschreibung. Nur Volutenrest.

Datierung: 6. Jahrhundert

Unpubliziert

K-14. Kämpferkapitell (Werkstück)

Marmor

Ort: LM, T. 2, Innenraum

Maße: Höhe 45, Breite $66 \mathrm{~cm}$

Beschreibung: Eine Nebenseite sichtbar. Kämpfer und obere Leiste schmucklos.

Datierung: 6. Jahrhundert

Unpubliziert

K-15. Kämpferkapitell

Marmor

Ort: BM, T. 10, Südflanke (Frühjahr 2001 verschollen)

Maße: Höhe 50, Breite $65 \mathrm{~cm}$

Beschreibung: Neben- und Hauptseite (rechts) sichtbar. Nebenseite schmucklos. Hauptseite schmucklose Fußleiste, Kämpfer mit einem reliefierten lateinischen Kreuz mit sich verbreiternden Enden, unterer und rechter Kreuzarm beschädigt.

Datierung: 6. Jahrhundert

Unpubliziert

Abb. 194

K-16. Kämpferkapitell (Werkstück)

Marmor

Ort: BM, K. 12/13, Feldseite

Maße: Höhe 28, Breite $72 \mathrm{~cm}$ (oben), $35 \mathrm{~cm}$ (Hals)

Beschreibung: Umgedreht eingemauert. Eine Langseite sichtbar. Fuß und Kämpfer schmucklos.

Datierung: 6. Jahrhundert

Unpubliziert

Abb. 202

K-17. Pfeilerkapitell mit Tierkampfgruppe und Löwenprotomen

Marmor

Ort: LM, T. 66, Südflanke

Maße: Höhe $50 \mathrm{~cm}$

Beschreibung: Eine Schmal- und eine Langseite mit hervortretenden Löwenprotomen an den Ecken, davon drei erhalten. Langseite Tierkampfszene. Hirsch von einem Löwen gerissen, flankiert von Akanthusranken. Schmalseite Löwe und Hirsch, beiderseits von Akanthusranken umgeben.

Datierung:-

Literatur: Meyer-Plath/Schneider, Landmauer, 69; Taf. 65, Abb. 1

K-18. Pfeilerkapitell mit Tierkampfgruppe und Löwenprotomen

Marmor

Ort: Benachbart zu K-17

Maße: Wie K-17

Beschreibung: Langseite wie K-17

Literatur: siehe K-17

Abb. 204 
K-19. Kämpferkapitell

Marmor

Ort: BM, K. 12/13, Feldseite

Maße: Höhe 31, Breite $31 \mathrm{~cm}$ (oben), $17 \mathrm{~cm}$ (Hals)

Beschreibung: Halsring mit Taustab. Kapitellkörper mit dreilappigen, schmalen Akanthusblättern, die sich durch Spitzenberührung zusammenschließen. Schmuckloser obere Leiste.

Datierung: Mittelbyzantinisch

Unpubliziert

Abb. 202

K-20. Kämpfer

Marmor

Ort, SM, T. 36, Seeseite

Maße: Hauptseite Höhe 36, Breite $142 \mathrm{~cm} /$ Nebenseite Breite $160 \mathrm{~cm}$

Beschreibung: Eine Haupt- und eine Nebenseite sichtbar. Hauptseite schmucklos. Nebenseite Fußleiste mit Eierstab, Körper mit stark hervortretendem mittleren Teil, dekoriert mit fünflappigem Akanthusblatt. Obere Leiste schmucklos.

Datierung: 5./6. Jahrhundert

Unpubliziert

\subsection{Tragende Elemente (Säulenschäfte, Säulenbasen und Postamente)}

TE-1. Runde Säulenbasis

Marmor

Ort: LM, T. 2, Innenraum

Maße: Höhe 35, Durchmesser $80 \mathrm{~cm}$

Beschreibung: Rechteckige Plinthe, Torus und Schaftring

Datierung: 5./6. Jahrhundert

Unpubliziert

TE-2. Runde Säulenbasis

Marmor

Ort: LM, benachbart zu TE-1

Maße: Höhe 35, Durchmesser $83 \mathrm{~cm}$

Beschreibung: Rechteckige Plinthe, Torus, Schaftsring

Datierung: 5./6. Jahrhundert

Unpubliziert

TE-3. Runde Säulenbasis

Marmor

Ort: SM, K. 81/82, Seeseite

Maße: Höhe 35, Durchmesser $80 \mathrm{~cm}$

Beschreibung: Torus, Trochillus, Torus, Schaftring

Datierung: 5./6. Jahrhundert

Unpubliziert

TE-4. Rundes Postament

Marmor

Ort: LM, Vorwerk des Goldenen Tors (Vortor), Nordseite 
Maße: Höhe ca. 150, Durchmesser ca. $80 \mathrm{~cm}$

Beschreibung: Dreistufige Fußleiste, hoher Körper, Trennleiste, angearbeitete attische Basis aus Torus, Trochillus und zweifach profiliertem Torus

Datierung:-

Literatur, Meyer-Plath/Schneider, Landmauer, 56; Dark/Özgümüş, Rescue, 23, Abb. 7 und 123.

TE-5. Würfelpostament

Marmor

Ort: LM, Vorwerk des Goldenen Tors, Nordseite

Maße: 70 × $70 \mathrm{~cm}$

Beschreibung: Vom Sockel nur obere Leiste erhalten. Postament an allen vier Seiten zwei quadratische Felder mit umlaufender Rahmenleiste, in den vertieften Feldern je ein Rundschild mit profiliertem Rahmen und Buckel im Zentrum; darüber durch Ritzlinie markierte Plinthe. Basis aus flachem Torus, Trochillus und doppeltem Torus.

Datierung: 5. Jahrhundert

Literatur: Strzygowski, Goldenes Thor, 19

Abb. 7, 124

TE-6. Würfelpostament

Marmor

Ort: LM, Vorwerk des Goldenen Tors, Südseite

Wie TE-5

Abb. 8, 124

TE-7. Postament mit Rautenmuster

Marmor

Ort: LM, K. 64/65, Stadtseite

Maße konnten nicht aufgenommen werden

Beschreibung: Eine Seite sichtbar. Umrahmt mit einer glatten Leiste. In der Mitte reliefierte Raute mit einem mittleren Kreuz. An drei Rautenspitzen je ein Herzblatt, unten Stengel mit Blatt unter der Rautenspitze.

Datierung: 5. Jahrhundert

Unpubliziert

TE-8. Spiralsäule

Marmor

Ort: GHM, Sepetçiler Köşkü, Westseite

Maße: $97 \mathrm{~cm}$

Beschreibung. Spiralsäule. Horizontal eingemauert.

Datierung: 5. Jahrhundert

Literatur: Tezcan, Topkap1, Abb. 16.

\subsection{Konsolen}

KO-1. Konsole mit Pfeifenmuster

Marmor

Ort: SM, K. 7/8, Seeseite

Maße: Höhe 44, Breite 51, Länge $120 \mathrm{~cm}$ 
Beschreibung: Eine Schmal- und eine Langseite sichtbar. Die nach außen abgerundete Schmalseite mit Pfeifenmuster und Lanzettblättern dazwischen. Beschädigte Kopfleiste. Grobe Langseite.

Datierung: 5./6. Jahrhundert

Literatur: Tezcan, Topkap1, 47, Abb. 33.

KO-2. Konsolbalken mit Adler

Marmor

Ort: SM, K. 9/10, Seeseite

Maße: Höhe 30, Länge $180 \mathrm{~cm}$

Beschreibung: Die Hälfe der Kopfseite und die Langseite sichtbar. Nach innen gerundete Kopfseite mit reliefiertem und geschupptem Flügel eines Adlers, schmucklose Kopfleiste.

Datierung: 5/6. Jahrhundert

Unpubliziert

Abb. 207

KO-3. Konsolbalken mit Stierkopf

Marmor

Ort: LM, T. 63, Vordere Ecken des Polygons

Maße konnten nicht aufgenommen werden

Beschreibung: Kopfseite und eine Langseite sichtbar. An der Schmalseite überhängender Stierkopf. Auf der Langseite profilierter Rechteckrahmen mit einem liegenden lateinischen Kreuz mit sich verbreiternden Enden. Rechts daneben geglättete Partie. Der ursprünglich in die Wand eingelassene Teil der Langseite mit Werkspuren.

Datierung: 5/6 Jahrhundert

Literatur: Meyer-Plath/Schneider, Landmauer, 78.

KO-4. Konsolbalken mit Löwenkopf

Marmor

Ort: Wie KO-3

Maße: Siehe KO-3

Beschreibung: Kopfseite und eine Langseite sichtbar. An der Kopfseite überhängender Löwenkopf. Auf der Langseite zwei profilierte Rechteckrahmen mit je einem liegenden lateinischen Kreuz mit sich verbreiternden Enden. Ein schmales geglättetes Feld, der ursprünglich in die Wand eingelassene Teil grob behauen, danach wieder ein glatter Teil. Datierung: Wie KO-3

Literatur: Wie KO-3

Abb. 191

KO-5. Konsole mit Pfeifenmuster

Marmor

Ort: LM, T. 26, Westfront

Maße: Höhe 29, Breite $28 \mathrm{~cm}$

Beschreibung: Langseite und Schmalseite sichtbar. Die Schmalseite nach innen gerundet, darauf vier Pfeifen, die vortretende Kopfleiste beschädigt.

Datierung: 5./6. Jahrhundert

Literatur: Meyer-Plath/Schneider, Landmauer, 75.

KO-6. Fragment einer Konsole mit Adler und Kreuz

Marmor

Ort: BM, T. 6, Südseite 
Maße: Höhe 60, Länge $200 \mathrm{~cm}$

Beschreibung: Umgekehrt eingemauert. Langseite sichtbar. Zwei hochrechteckige Felder umgeben von einem zweifach profilierten Rahmen. Im linken Feld griechisches Kreuz mit sich verbreiternden Enden, diagonal von einer Lanze durchkreuzt, der untere Kreuzarm beschädigt. Im rechten Feld Adler mit ausgebreiteten Flügeln und nach rechts gewendetem Kopf. Ein drittes Feld rechts mit einer nicht mehr erkennbaren Füllung. Unterer Rand beschädigt.

Datierung: 6. Jahrhundert

Unpubliziert

Abb. 194

KO-7. Konsole mit Akanthuswirbelranke und Kantharos

\section{Marmor}

Ort: Kurtine zwischen TS/TS-Nordturm

Maße: Höhe 58. Länge $215 \mathrm{~cm}$

Beschreibung: Umgekehrt eingemauert. Langseite und Kopf teilweise sichtbar. Größter Teil der Langseite grob behauen. Auf der Wange des Konsolenkopfes Akanthuswirbelranken mit nach innen gewendeten Blattwedeln und Dreiblättern in den Zwickeln. Vordere Schmalseite nach außen gerundet, darauf weitere Blätter und Trennleiste. Auf der Langseite anschließend hochrechteckiges, gerahmtes Bildfeld mit einem Kantharos. Zwei dreizackige Akanthusblätter fallen aus dem Kantharos seitlich nach unten, ein weiteres Dreiblatt ragt aus der Kantharosöffnung heraus. Links und oben zweilappiges Akanthusblatt mit zwei bzw. drei Zacken. Erstaufnahme Herbst 1996, seit Sommer 2000 unter der Aufschüttung verborgen.

Datierung: 6. Jahrhundert

Literatur: Meyer-Plath/Schneider, Landmauer, 65, Abb. 5.

KO-8. Akanthuskonsole

Marmor

Ort: LM, Vorwerk des Goldenen Tors, Feldseite, Süden

Maße: Höhe 35, Breite $30 \mathrm{~cm}$

Beschreibung: Auf der unteren Kehle der Kopfseite neunlappiger, großgezahnter Akanthus, darüber glatte Leiste. Die Nebenseiten durch eine Leiste in zwei Felder unterteilt. Das obere Feld mit einem füllhornähnlichen Blattkelch und davon ausgehenden Dreiblättern, das untere Feld mit akanthisierenden Ranken dekoriert.

Datierung: 5./6. Jahrhundert

Literatur Strzygowski, Goldenes Thor, 22-23, Abb. 15.

Abb. 185

KO-9. Akanthuskonsole

Marmor

Ort: siehe $\mathrm{KO}-8$

Maße: siehe $\mathrm{KO}-8$

Beschreibung: Auf der unteren Kehle der Kopfseite Akanthusblatt, darüber glatte Leiste. Die Nebenseiten sind durch eine horizontale Leiste in zwei Felder unterteilt. Im oberen

Feld eine Wellenranke, im unteren Feld drei Blätter.

Datierung: Wie KO-8

Literatur: Wie KO-8

KO-10. Akanthuskonsole

Marmor

Ort: siehe KO-8 
Maße: siehe KO-8

Beschreibung: Konsolenkopf abgebrochen. Die Nebenseiten sind durch eine horizontale Leiste in zwei Felder unterteilt. Im oberen Feld ein einfacher Lorbeerblattstab, im unteren Feld sich einrollende Ranken einer Palmette?

Datierung: Wie KO-8

Literatur: Wie KO-8

KO-11. Fragment einer Konsole mit Doppelranke

Marmor

Ort: LM, T. 84, Westseite

Maße: Höhe 23, Breite $400 \mathrm{~cm}$

Beschreibung: Langseite mit Ansatz des im ursprünglichen Mauerverband verborgenden Endstücks sichtbar. Oben und unten Rahmenleisten. Innenfeld mit Doppelranke aus zwei einander durchdringenden, zweisträhnigen Wellenranken, die in Blattformen auslaufen und vier Ellipsen bilden, die mit je einer liegenden, mandelförmigen Perle gefüllt sind. In den Zwickeln stehende, mandelförmige Perlen. Die Ranke schließt rechts an ein Füllhorn an, die äußeren Zwickel mit Herzblattzweigen dekoriert. Das Bildfeld rechts abgeschrägt. Der Konsolenkopf undekoriert, Werkspuren.

Datierung: 5./6. Jahrhundert

Literatur: Meyer-Plath/Schneider, Landmauer, 65, Abb. 3.

KO-12. Fragment einer Konsole mit Wellenranke

Marmor

Ort: LM, T. 84, Westseite

Maße: Höhe 23, Breite $210 \mathrm{~cm}$

Beschreibung: Längsseite sichtbar. Oben und unten Rahmenleisten. Im Innenfeld unregelmäßige Wellenranke mit seitlich abzweigenden Lanzettblättern, die in zwei Hebungen und zwei Senkungen nach links verläuft und bis zur ersten Hebung zurückkehrt, wo sie mit drei Lanzettblättern endet.

Datierung: 5./6. Jahrhundert

Literatur: Meyer-Plath/Schneider, Landmauer, 65, Abb. 3.

Abb. 110

KO-13 Fragment einer Konsole mit Wellenranke

Marmor

Ort: Wie KO-12

Maße: Höhe 23, Breite $100 \mathrm{~cm}$

Beschreibung: Endstück, fast identisch mit dem rechten Teil von KO-12, nur endet die

Ranke hier über der ersten Senkung.

Datierung: 5./6. Jahrhundert

Unpubliziert

\subsection{Gesimse, Friese, Architrave}

GFA-1. Glattes Konsolgesims

Marmor

Ort: LM, Vorwerk des Goldenen Tors, Feldseite, Süden

Maße: insgesamt ca. 3,5 m lang

Beschreibung: Verkröpfter Teil profiliert 
Datierung: 5/6. Jahrhundert

Literatur: Strzygowski, Goldenes Thor, 23, Abb. 15.

GFA-2. Verkröpfter Fries mit Akanthusblättern

Marmor

Ort: LM, Vorwerk des Goldenen Tors, Feldseite, Süden

Maße: ca. $3 \mathrm{~m}$

Beschreibung: Zahnschnitt, Wulst, durchgehendes, fünflappiges Akanthusband. Akanthen schließen sich durch Spitzenberührung zusammen. Glatte Kopfleiste. Am verkröpften Teil Zahnschnitt, Trennleiste, Kehle, Trennleiste, Sägezahnband, Trennleiste, darauf Wiederholung der Abfolge wie am Gesims. Auf der Auflagefläche des verkröpften Teils zwei rechteckige Einlaßlöcher. Auf der Oberseite zwei lateinische Kreuze auf einer Sphaira stehend, die oberen Kreuzarme beschnitten. (Die oberen Arme der beiden Kreuze wurden während der Herstellung des Gesimses für den Zweck der Wiederverwendung abgeschnitten.)

Datierung: Frühestens 6. Jahrhundert

Literatur: Strzygowski, Goldenes Thor, 23, Abb. 16; Macridy/Casson, Excavations, Taf. 38, Abb. 4.

Abb. 8, 205

GFA-3. Friesfragment mit Akanthusblattreihe

Marmor

Ort: SM, vor dem Bukoleon-Palast, sog. Seepforte, linke Seeseite

Maße: Höhe 50, Tiefe $82 \mathrm{~cm}$ (jeweils)

Beschreibug: In mehrere Blöcke zerschnitten (Mamboury), in sechs Lagen übereinander gestapelt und im rechten Winkel angeordnet. Davon sind nur noch vier Blöcke der oberen drei Lagen sichtbar. Dreistufige Fußleiste, die mittlere Stufe mit Astragal. Blattstab aus Akanthus mit fünflappigen Blättern. Glatte Trennleiste. Breiterer Fries mit bewegten Akanthusranken, im Zentrum eines Segments lateinisches Kreuz mit sich verbreiternden Enden. Trennleiste, darüber abgearbeitetes oder unvollendetes Feld. Am dritten Stück von oben lesbisches Kyma über dem bewegten Akanthus. Das vierte Stück von oben ist an der linken Schmalseite abgerundet und mit Ornamenten besetzt. Die Unterseiten sind mit rechteckigen Feldern (Soffitten) dekoriert.

Datierung: 6. Jahrhundert

Literatur: Mamboury/Wiegand, Kaiserpaläste, 6 ff., Taf. XV, XVI, XVII, XVIII; Mango, Spolia, 653, Abb. 7.

GFA-4. Gesimsfragment mit Kreuz und Palmetten

Marmor

Ort: LM, K. 80/81, Feldseite

Maße: Breite 50, Höhe $8 \mathrm{~cm}$

Beschreibung: Ragt aus dem Mauerverband leicht heraus. Seitlich abgeschnitten. Untere Leiste nicht sichtbar. Schräge Stirn- und Kopfleiste. Auf der Stirnleiste im Wechsel miteinander verbundene fünfblättrige Palmetten und griechische Kreuze mit sich verbreiternden Enden und eingezeichnetem Kreuz, alles in „umgekehrtem“ Relief.

Datierung: 10. Jahrhundert

Unpubliziert

GFA-5. Vier Fragmente eines Frieses mit Kreisbandgeflecht

Marmor

Ort: LM, T. 83, Westseite 
Maße: Teil 1 Höhe 32, Länge ca. $125 \mathrm{~cm}$; Teil 2 Höhe 32, Länge ca. $70 \mathrm{~cm}$; Teil 3 Höhe 32, Länge ca. $230 \mathrm{~cm}$; Teil 4 Höhe 32, Länge ca. $90 \mathrm{~cm}$

Beschreibung: In vier anpassende Teile zerlegt. Nur die Vorderseiten sichtbar.

Zweifach profilierte Kopf- und Fußleiste, jedoch die Fußleiste etwas breiter. Auch das Endstück (Teil 1) ist zweifach profiliert bzw. (Teil 4) läuft mit einem Halbkreis stumpf aus. Im Innenfeld doppelsträhnige, untereinander durch Knoten verbundene Kreismedaillons, die abwechselnd mit Vögeln zwischen Zweigen, Blütenrosetten (3 Typen) und lateinischen Kreuzen zwischen Akanthusblättern (Lebensbaumkreuz) gefüllt sind. Oberund unterhalb der Knoten je ein dreifingriges Blatt als Zwickelfüllung.

Datierung: 5./6. Jahrhundert

Literatur, Meyer-Plath/Schneider, Landmauer, 65, Abb. 3; Krumeich, Bauskulptur, 198, Abb. 33.

Abb. 195

GFA-6. Fragment eines Frieses mit Kreisbandgeflecht

Marmor

Ort: LM, T. 83, Westseite (zwischen Teil 3 und Teil 4 von GFA-5)

Maße: Höhe 32, Länge ca. $237 \mathrm{~cm}$

Beschreibung: Umgekehrt eingesetzt. Gleiches Dekorationsschema wie GFA-5, jedoch die Blätter ober- und unterhalb der Knoten mit tiefen Bohrlöchern. Die Richtung der Knoten ist umgekehrt.

Datierung: Wie GFA-5

Literatur: Wie GFA-5

Abb. 196

GFA-7. Gesims mit Pfeifenfries

Marmor

Ort: SM, K. 11/15, Seeseite

Maße konnten nicht aufgenommen werden

Beschreibung: In vier Teile zerbrochen. Langseiten sichtbar. Einfach profilierte Fußleiste, S-förmige Kehle. Pfeifenreihe, zwischen den Pfeifen Lanzettblätter, am Fuß der Pfeifen ein halbkreisförmiger Knauf. Kopfleiste beschädigt. Standfläche zum Teil sichtbar.

Datierung: 5./6. Jahrhundert

Literatur: Demangel/Mamboury, Manganes, 77, Abb. 75; 82, Zeichnung 79.

Abb. 206

GFA-8. Gesims mit Pfeifenfries

Marmor

Ort: Mermerkule, Seeseite, rechte Ecke

Maße: Höhe 13, Länge $50 \mathrm{~cm}$

Beschreibung: Langseite sichtbar. Fußleiste, darüber Astragal, S-förmige Kehle mit Pfeifenfries, dazwischen Lanzettblätter, am Fuß der Pfeifen gerahmte Knäufe. Kopfleiste beschädigt.

Datierung: 5./6. Jahrhundert

Unpubliziert

Abb. 132

GFA-9. Gesims mit Pfeifenfries

Marmor

Ort: LM, Vorwerk des Goldenen Tors, Feldseite, Süden

Maße: Höhe 5, Länge $75 \mathrm{~cm}$ 
Beschreibung: Langseite sichtbar. Fußleiste, darüber Astragal, S-förmige Kehle mit Pfeifenfries, dazwischen Lanzettblätter. Am Fuß der Pfeifen gerahmte Knäufe. Pfeifenbögen und Fußleiste beschädigt.

Datierung: 5./6. Jahrhundert

Unpubliziert

GFA-10. Fragment eines Gesimses mit Lotus-Palmetten-Fries

Marmor

Ort: SM, K. 80/81, Feldseite

Maße: Höhe 12, Länge $67 \mathrm{~cm}$

Beschreibung: Umgekehrt eingemauert. Vorderseite sichtbar. Kopf- und Fußleiste leicht beschädigt. In einer Reihe Lotus-Palmetten-Fries. Die äußeren Blätter des Lotus schließen sich durch Spitzenberührung zusammen und bilden für die darunter stehenden Palmetten einen Bogen.

Datierung: 10. bis 12. Jahrhundert

Unpubliziert

GFA-11. Fragment eines Gesimses mit Lotus-Palmetten-Fries bzw. Lotus-PalmettenWellenranken

\section{Marmor}

Ort: Mermerkule, Hof

Maße konnten nicht aufgenommen werden

Beschreibung: In mehrere Teile zerlegt, einige wohl verloren. Darunter auch L-förmige Eckgesimse. Eine Reihe Lotusblätter und Palmetten (in einem Stück mit Wellenranken). Die äußeren Blätter des Lotus schließen sich durch Spitzenberührung zusammen und bilden für die darauf stehende Palmette einen Bogen.

Datierung: 10. bis 12. Jahrhundert

Literatur, Peschlow, Mermerkule II, 390-391., Abb. 10-16.

Abb. 135

GFA-12. Fries mit Rautenband

\section{Marmor}

Ort: LM, T. 84, Südseite

Maße: Höhe 22, Breite $47 \mathrm{~cm}$

Beschreibung: Teil der Lang- und Rückseite sichtbar. Links eine breite Rahmenleiste. Schmalere Kopf- und Fußleiste. Mit je einem Vierblatt gefülltes doppelstreifiges Rautengitter. Die äußersten Zwickel mit Dreiblatt. Einlassungsflügel der Rückseite ist hinter dem Gesims oben und unten zurückgezogen.

Datierung: 5./6. Jahrhundert

Unpubliziert

Abb. 198

GFA-13. Gesims mit Palmetten

Marmor

Ort: SM, T. 102

Maße: Höhe 25, Breite $145 \mathrm{~cm}$

Beschreibung: Zwei Seiten sichtbar. Bogenförmig angeordnete Blattranken, gefüllt mit birnenartigen Früchten, die mit kurzen Stielen an den oberen Rankenbögen hängen Datierung: 10 bis 12. Jahrhundert

Unpubliziert 
GFA-14. Zwei Blöcke eines Gesimses mit Psalm-Inschrift

Marmor

Ort: SM, K. 36/37, Seeseite (Sergios und Bakchos-Poterne)

Maße: Linkes Stück Länge 100, Breite 32, Tiefe $41 \mathrm{~cm}$; rechtes Stück Länge 100, Breite 32, Tiefe $41 \mathrm{~cm}$

Beschreibung: Architrav mit drei Faszien, Fries mit Inschrift. Zu Beginn des 20. Jahrhunderts, als der größte Teil der Poterne noch sichtbar war, wurde die Inschrift noch vollständig gelesen.

Datierung: 6. Jahrhundert (?)

Literatur: Mordtmann, Esquisse, 21; Van Millingen, Walls, 262; Dirimtekin, Marmara, 32; Mango, Inscriptions, 55.

GFA-15 Gesims mit Palaiologen-Monogramm

Marmor

Ort: Mermerkule

Maße konnten nicht aufgenommen werden

Beschreibung: Kreisbänder mit Blüten, Palaiologen-Monogramm und Doppelhastenmotiv gefüllt

Datierung: Palaiologisch

Literatur, Peschlow, Mermerkule I, 91-96, Abb. 3; Peschlow, Mermerkule II, 392, Abb. 17.

Abb. 134

GFA-16 Gesims mit Birnenranke

Marmor

Ort: SM, T. 82, Seeseite

Maße: Höhe 25, Breite $145 \mathrm{~cm}$

Beschreibung: Zwei Seiten sichtbar. Bogenförmig angeordneter Blattdekor, gefüllt mit birnenartigen Früchten, die mit kurzen Stielen an den oberen Rankenbögen hängen. Datierung: 6. Jahrhundert

Unpubliziert

Abb. 211

GFA-17 Gesimsfragment

Marmor

Ort: LM, T. 61, Westseite

Maße konnten nicht aufgenommen werden

Beschreibung: Getreppte Fußleiste, Astragal, Lotospalmette, Kymation

Umpubliziert

Abb. 197

\subsection{Türrahmen}

T-1. Türgewände mit Monogramm

Marmor

Ort: SM, vor Bukoleon-Palast, sog. Seepforte

Maße: Höhe 50, Tiefe. $80 \mathrm{~cm}$ (jeweils)

Beschreibung: In drei Lagen übereinander gestapelt und im rechten Winkel angeordnet, davon gehören die Teile der beiden oberen Lagen zum Portal. Dreistufiges Laibungsprofil (Leiste, halbrunde Kehle, Leiste), aufgelegte Trennwulst (Rundstab), flache Kehle 
mit schmaler Trennleiste, schräge Außenfläche mit lesbischem Kyma und schmaler Leiste. Auf dem Sturz in der Mitte oberhalb des Rundstabes ein gerahmtes Medaillon mit Monogramm Justinians I. Monogramm und Schmuckfeld seit 2000 stark beschädigt.

Datierung: 6. Jahrhundert

Literatur: Mamboury/Wiegand, Kaiserpaläste, 6-7, Taf. XV, XVI, XVII; Mango, Spolia, 653, Abb. 8.

\section{T-2. Türsturz mit Psalm-Inschrift}

Marmor

Ort: SM, K. 15/16, Stadtseite (sog. Hodegetria-Tor)

Maße: Höhe 33, Länge 129 und $164 \mathrm{~cm}$

Beschreibung: In zwei Teile zerbrochen. Nur die Vorderseiten sichtbar. Zweistufige Leiste, S-förmige Kehle, darauf gerahmtes Monogramm, von dem je ein Schleifenband nach links und nach rechts ausgeht und in einem Herzblatt endet, auf dem jeweils ein lateinisches Kreuz mit sich verbreiternden Enden steht. Kopfleiste mit einer PsalmInschrift (Psalm 117 [118], 19).

Datierung: 5./6. Jahrhundert

Literatur: Demangel/Mamboury, Manganes, 76, Abb. 80, 81; 78, Abb. 84.

T-3. Türsturz

Marmor

Ort: Vorwerk des Goldenen Tors, Feldseite, Portal

Maße konnten nicht aufgenommen werden

Beschreibung: Obere Leiste, S-förmige Kehle. Christogramm mit Schleifen

Datierung: 5./6. Jahrhundert

Literatur: Strzygowski, Goldenes Thor,17-18; Meyer-Plath/Schneider, Landmauer, Taf. 29a.

Abb. 124

\subsection{Platten und Schrankenplatten}

P-1. Fragment einer Platte mit Rapportmuster aus Oktogonen

Marmor

Ort: SM, K. 11/15 (dazwischen befinden sich heute keine Türme mehr), Seeseite

Maße: Höhe 75, Breite $50 \mathrm{~cm}$

Beschreibung: Seitlich eingemauert. Vorderseite sichtbar. Links dreifach profiliert. Inneres Feld Rapportmuster aus doppelt gerahmten Oktogonen mit nach innen gerundeten Diagonalseiten, zwischen den Oktogonen doppelt gerahmte Kreise.

Datierung: 5./6. Jahrhundert

Literatur: Tezcan, Topkap1, 47, Abb. 35.

P-2. Fragment einer Platte mit Rapportmuster aus Stäbchen

Marmor

Ort: SM, K. 11/15, Seeseite

Maße: Höhe 80, Breite $50 \mathrm{~cm}$

Beschreibung: Seitlich eingemauert. Vorderseite sichtbar. Links und unten umlaufende Randzone und zweifach profilierte Randseite. Im Feld Rapportmuster aus rechtwinklig angeordneten zweisträhnigen Stäbchen.

Datierung: 5./6. Jahrhundert

Literatur: Tezcan, Topkap1, 47, Abb. 36. 
P-3. Fragment einer Platte mit Rautenmuster

Marmor

Ort: SM, T. 36, Seeseite

Maße: Breite 41, L. $92 \mathrm{~cm}$

Beschreibung: Platte in den Mauerverband eingeschoben. Nur eine Seite schwer erkennbar. Konzentrisch reliefierte Rechtecke und reliefierte Rauten.

Datierung: 5./6. Jahrhundert

Unpubliziert

P-4. Fragment einer Platte mit Rapportmuster aus Rauten

Marmor

Ort: LM, T. 36a, Westseite

Maße: Höhe ca. 80 , Breite ca. $80 \mathrm{~cm}$

Beschreibung: Teil der Vorderseite sichtbar. Einfache Randleiste. Im inneren Feld reliefierter Rautenrapport, gefüllt mit Muschel und Fisch.

Datierung: 5./6. Jahrhundert

Unpubliziert

P-5. Fragment einer Platte mit Kreuz

Marmor

Ort: LM, K. 51/52, Feldseite

Maße: Breite $80 \mathrm{~cm}$

Beschreibung: Erhalten ist die rechte Randleiste und der untere Arm eines reliefierten Kreuzes mit sich verbreiternden Enden im inneren Feld. Untere Sockelzone und linke Randleiste abgearbeitet

Datierung: 6. Jahrhundert

Literatur: Meyer-Plath/Schneider, Landmauer, 82.

Abb. 190

P-6. Fragment einer Schrankenplatte mit Kranz und Staurogramm

Marmor

Ort: Nähe Belgratkapı, liegt frei zwischen Vor- und Hauptmauer

Maße: Höhe 95, Breite $71 \mathrm{~cm}$

Beschreibung: Linke Hälfte weggebrochen. Zweifach gestufte Rahmenleiste. Im inneren Feld Bandgeflecht aus doppelsträhnigen mit Akanthusblüten gefüllten Vierpässen, davon zwei und ein schmuckloser zweisträhniger Zweipaß erhalten. Im Zentrum Lorbeerkranz, gefüllt mit einem Staurogramm und den apokalyptischen Buchstaben Alpha und Omega, vom Staurogramm ein Rest des Querarms, der Rho-Schlinge und das Omega erhalten. Die Schleife geht vermutlich in den unteren Vierpaß über. Rückseite mit zweifach gestufter Randleiste und schmucklosem inneren Feld.

Datierung: 10./11. Jahrhundert

Unpubliziert

P-7. Platte mit Kreisen

Marmor

Ort: SM, K. 7/8, Seeseite

Maße konnten nicht aufgenommen werden

Beschreibung: Ragt aus dem Mauerverband hervor. Schmucklose Rückseite. Auf der Vorderseite durch dreistreifiges Band verbundene, dreistreifige Kreise.

Datierung: 10./11. Jahrhundert

Literatur: Unpubliziert 
P-8. Platte mit Adler

Marmor

Ort: LM, Yedikule, oberhalb des Yedikulekapısı, Stadtseite

Maße konnten nicht aufgenommen werden, ca. 80 × $80 \mathrm{~cm}$

Beschreibung: Vordere Seite sichtbar. Fußleiste abgeschnitten. Schmale seitliche Randleisten, breitere Kopfleiste. Im inneren Feld ein reliefierter Adler mit ausgebreiteten Flügeln und nach rechts gewendetem Kopf. Das Gefieder teils reliefiert, teils gebohrt, die Flügeln sind durch ein gebohrtes Band zweigeteilt, die obere Hälfte der Flügeln schraffiert, die untere Hälfte mit vertikal linierten und schraffierten Federn.

Datierung: 10. Jahrhundert

Literatur: Meyer-Plath/Schneider, Landmauer, 63.

Abb. 10

P-9. Platte mit Spiralranken

Marmor

Ort: BM, K. 8/9, Feldseite

Maße konnten nicht aufgenommen werden

Beschreibung: Linke untere Ecke, profilierter Rahmen, fortlaufende Spiralranke mit davon ausgehenden dreiblättrigen Palmetten.

Datierung: Römische Kaiserzeit

Literatur: Unpubliziert

Abb. 192

\subsection{Schranken- und Fensterpfeiler}

PF-1. Pfeiler mit floralem Dekor

Marmor

Ort: SM, K. 7/8, Seeseite

Maße konnten nicht aufgenommen werden

Beschreibung: Ragt, von einem Konsolenkopf unterstützt, aus dem Mauerverband hervor. Auf einer Seite hohe seitliche Leisten und schwer erkennbare Ranken im Mittelstreifen. Nuten an beiden Seiten und Dübelloch auf der Standfläche.

Datierung: -

Literatur: Tezcan, Topkap1, Abb. 29.

PF-2. Pfeiler mit Blattdekor und Granatapfel

Marmor

Ort: SM, K. 21/22, Seeseite

Maße: Höhe 105, Breite $30 \mathrm{~cm}$

Beschreibung: Vordere Seite mit zwei Schmuckfeldern ganz und Nebenseite mit durchgehender Nut teilweise sichtbar. Auf der Vorderseite durch einen breiten Steg getrennt zwei rechteckige Bildfelder mit versenktem Rahmen, der zwei Bildfelder umgibt. An den äußeren Ecken der inneren Rahmen je ein Lanzettblatt. Die beiden durch eine horizontale Leiste getrennten Innenfelder unten mit je einem doppellappigen Akanthusblatt mit seitlich abzweigenden Voluten, oben mit einem Vierpaß mit Mittelperle und einem Granatapfel darüber gefüllt.

Datierung: 5. Jahrhundert

Literatur: Tezcan, Topkap1, 48, Abb. 42. 
PF-3. Pfeiler mit Doppelblattstab

Marmor

Ort: LM, Goldenes Tor, nördlicher Durchgang, Feldseite

Maße: Höhe 91, Breite $12 \mathrm{~cm}$

Beschreibung: Eine Langseite sichtbar. Seitliche Rahmenleisten. Im inneren Feld aufsteigender, doppelstreifiger Blattstab mit gegenständigen Blättern.

Datierung:-

Unpubliziert

Abb. 200

PF-4. Pfeilerfragment mit umlaufender Herzblattranke

Marmor

Ort: SM, K. 4/5 Seeseite

Maße: Höhe 125, Breite $45 \mathrm{~cm}$

Beschreibung: Eine Langseite sichtbar. Schmucklose Kopfleiste, seitliche Trennleisten.

Eine reliefierte Herzblattranke umgibt das schmucklose innere Feld.

Datierung: 6. Jahrhundert

Literatur: Demangel/Mamboury, Manganes, 9, Abb. 5; Tezcan, Topkapı, 45, Abb. 22.

PF-5. Pfeiler mit Akanthusblättern

Marmor

Ort: Mermerkule

Maße: Höhe 120, Breite $40 \mathrm{~cm}$

Beschreibung: Eine Langseite sichtbar. Dreifach profilierte Fußleiste, schmuckloser Körper, Trennleiste, Schlußband mit einem fünflappigen Akanthusblatt, flankiert von zwei Akanthus-Halbblättern.

Datierung: Frühbyzantinisch

Unpubliziert

PF-6. Pfeiler mit graviertem Kreuz

Marmor

Ort: LM, T. 5, Südflanke

Maße: Höhe 118, Breite 48, Tiefe $33 \mathrm{~cm}$

Beschreibung: Eine Langseite und die Standfläche mit einem Einlassungsloch sichtbar. An der oberen Partie der Langseite eingraviertes lateinisches Kreuz mit sich verbreiternden Enden.

Datierung: nach 600

Unpubliziert

PF-7. Pfeiler mit Kreuz und Globus

Marmor

Ort: LM, T. 24, Südflanke

Maße konnten nicht aufgenommen werden

Beschreibung: Eine Langseite und Standfläche sichtbar. Schmucklose Fußleiste, obere Partie mit einem auf einem Globus stehenden lateinischen Kreuz mit sich verbreiternden Enden in versenktem Relief, Trennleiste, Kopfleiste.

Datierung: 6. Jahrhundert

Literatur: Meyer-Plath/Schneider, Landmauer, 75.

Abb. 189 


\subsection{Kaiserporträts}

KP-1. Kaiserbüste

Marmor

Ort: BM, K. 11/12, rechts (sog. Gyrolimne-Tor)

Maße konnten nicht aufgenommen werden

Beschreibung: Büstenteil mit Perlenkragen geschmückt.

Datierung: 6. Jahrhundert

Literatur: Meyer-Plath/Schneider, Landmauer, 114.

Abb. 158, 159b

KP-2. Kaiserbüste

Marmor

Ort: Wie KP-1, Mitte

Maße konnten nicht aufgenommen werden

Beschreibung: Büstenteil mit Perlenkragen und Pendilien geschmückt.

Datierung: Wie KP-1

Literatur: Van Millingen, Walls, VIII (Abb.); Schneider, Landmauer, 114.

Abb. 158, 159a und c

KP-3. Kaiserbüste

Marmor

Ort: Wie KP-1, links

Maße konnten nicht aufgenommen werden

Beschreibung: sehr beschädigt

Datierung: Wie KP-1

Literatur: Meyer-Plath/Schneider, Landmauer, 114.

Abb. 158

\subsection{Sarkophagfragmente}

SAR-1. Zwei Akrotere

Kalkstein

Ort: SM, T. 17, Nordseite

Maße: je Höhe 27, je Breite $32 \mathrm{~cm}$

Beschreibung: Zwei männliche Büsten mit bis zum Kinn herabreichendem langem Haar, bekleidet mit Tunika (?) (der linke bereits im Jahre 2000 beschädigt)

Datierung: 5. Jahrhundert

Literatur: Demangel/Mamboury, Manganes 82, Abb. 89; Tezcan, Topkap1, 47-48, Abb. 39; Asutay, Akroter, 25-31.

Abb. 209, 210

\subsection{Fragmente von Reparaturinschriften der Stadtmauern}

I-1. Fragment

Marmor

Ort: GHM, Sepetçiler Köşkü, Seeseite 
Maße: ca. $18 \mathrm{~cm}$

Beschreibung: Eingetiefte Buchstaben mit Einlassungslöchern, als POC + zu lesen.

Datierung: 9. Jahrhundert (Theophilos)

Literatur, Tezcan, Topkap1, 42, Abb. 18 (seitenverkehrt).

I-2. Fragment

Marmor

Ort: GHM, Sepetciler Köşkü, Seeseite

Maße: $13 \mathrm{~cm}$

Beschreibung: Reliefierte Buchstaben, als ПҮРГОС ЄЕОФІ $\Lambda$ Ү zu lesen.

Datierung: 9. Jahrhundert (Theophilos)

Literatur: Tezcan, Topkap1, 41-48; Abb. 17 (seitenverkehrt).

I-3. Fragment

Marmor

Ort: SM, T. 7, Seeseite

Maße: $18 \mathrm{~cm}$

Beschreibung: Eingetiefte Buchstaben mit Einlassungslöchern, als OPOC + zu lesen. Datierung: 9. Jahrhundert (Theophilos)

Literatur: Demangel/Mamboury, 11, Abb. 14; Curtis/Aristarchis, 28, Nr. 124; Dirimtekin, Marmara 62, Nr. 9; Tezcan, Topkap1, 46-47, Abb. 24.

I-4. Fragment

Marmor

Ort: SM, K. 9/10, Seeseite

Maße: $13 \mathrm{~cm}$

Beschreibung: Obere und untere Leiste stark beschädigt. Reliefierte Buchstaben, als $\omega \mathrm{P} \omega \mathrm{N}+$ zu lesen.

Literatur: Demangel/Mamboury, Manganes, 58, Abb. 59; Dirimtekin, Marmara, 63, Nr. 11; Mango, Inscriptions, 56, Nr. 26; Tezcan, Topkap1, 46-47, Abb. 32.

Datierung: 9. Jahrhundert (Theophilos)

I-5. Fragment

Marmor

Ort: SM, K. 9/10, Seeseite

Maße: $16 \mathrm{~cm}$

Beschreibung: Obere und untere Leiste beschädigt. Reliefartige Buchstaben, als AYTOKPA zu lesen.

Datierung: 9. Jahrhundert (Theophilos)

Literatur: Demangel/Mamboury, Manganes, 58, Abb. 58; Dirimtekin, Marmara, 63,

Nr. 10; Mango, Inscriptions, 56, Nr. 25; Tezcan, Topkapı, 47, Abb. 34.

I-6. Fragment

Marmor

Ort: LM, T. 5, Westseite

Maße: $18 \mathrm{~cm}$

Beschreibung: Eingravierte Buchstaben, als KAIK $\omega$ NC zu lesen.

Datierung: 8. Jahrhundert (Leon und Konstantin)

Literatur: Meyer-Plath/Schneider, Landmauer, 71 und 124.

Abb. 187

I-7. Fragment

Marmor 
Ort: LM, K. 80/81, Feldseite

Maße: $14 \mathrm{~cm}$

Beschreibung: Umgekehrt eingemauert. Obere und untere Leiste beschädigt. Reliefierte Buchstaben, als + ANEKAINICTI zu lesen.

Datierung: 15. Jahrhundert

Unpubliziert

Abb. 188

I-8. Fragment

Marmor

Ort: LM, K. 81/82, Stadtseite

Maße: ca. $14 \mathrm{~cm}$

Beschreibung: Reliefierte Buchstaben, als KRATOP zu lesen

Datierung: 9. Jahrhundert (Theophilos)

Literatur: Meyer-Plath/Schneider, Landmauer, 137, Nr. 47.

I-9.Fragment

Marmor

Ort: BM, T. 14, Westseite

Maße: ca. $14 \mathrm{~cm}$

Beschreibung: Ohne obere und untere Leiste. Eingetiefte Buchstaben mit Einlaßlöchern, als ОФІ $\Lambda$ OY zu lesen.

Datierung: 9. Jahrhundert (Theophilos)

Literatur: Paspatis, Meletai, 38; Meyer-Plath/Schneider, Landmauer, 141, Nr. 61.

I-10. Fragmente

Marmor

Ort: GHM, K. 11/12, Seeseite

Maße: $14 \mathrm{~cm}$

Beschreibung: Obere und untere Leiste beschädigt, als N X $\omega \mathrm{A}$ zu lesen.

Datierung: Jahrhundert (Theophilos)

Literatur: Dirimtekin, Haliç, 69, Abb. 43.

\subsection{Varia}

V-1. Fragment mit Akanthus

Marmor

Ort: LM, T. 19, Nordflanke

Maße: Höhe 39, Breite $27 \mathrm{~cm}$

Beschreibung: Seitlich eingemauert. Pfeilerrest und Teil des Kapitellkörpers (?), Trennleiste und Fuß (?). Kapitellkörper aus fünflappigen Akanthusblättern, Mittelrippe mit überfallendem Dreiblatt.

Datierung: 5./6. Jahrhundert

Unpubliziert

V-2. Block mit Kreuz

Marmor

Ort: SM, K. 94/95, Stadtseite

Maße: Höhe 12, Breite $46 \mathrm{~cm}$ 
Beschreibung: Schwach angedeutete obere Leiste. Rechts eingraviertes Kreuz mit sich verbreiternden Enden.

Datierung: -

Unpubliziert

V-3. Bogenrest

Marmor

Ort: BM, 14-Meter-Mauer, Stadtseite

Maße: Höhe 14, Breite $30 \mathrm{~cm}$

Beschreibung: Zweistufige Fußleiste, nach außen gerundetes breites Feld mit einem abgearbeiteten Knauf und Rest eines Rahmens, zweistufige Stirnleiste.

Datierung: -

Unpubliziert

V-4. Fragment einer Spiralsäule

Marmor

Ort: SM, K. 68/69, Stadtseite

Maße: Höhe 13, Breite $10 \mathrm{~cm}$

Beschreibung: Ragt aus dem Mauerverband heraus. Linker Teil gerundet und abgefast, rechter Teil mit vorgeblendeter Spiralsäule.

Datierung: 5. Jahrhundert

Unpubliziert

V-5. Fragment mit Fischgrätenmuster

Marmor

Ort: SM, K. 68/69, Langa Bostanı, Stadtseite

Maße: Höhe 13, Breite $31 \mathrm{~cm}$

Beschreibung: Zweifach profilierte Leiste, breiteres Feld mit Fischgrätenmuster, schmucklose Leiste.

Datierung: -

Unpubliziert

V-6. Fragment mit Reliefkreuz

Marmor

Ort: BM, K. 5/6

Maße konnten nicht aufgenommen werden

Beschreibung: Mittlerer Teil eines reliefierten Kreuzes.

Datierung: -

Unpubliziert

V-7. Gerundetes Stück mit Kreuz

Marmor

Ort: SM, T. 4, Seeseite

Maße konnten nicht aufgenommen werden

Beschreibung: Seitlich eingemauert. Umrahmtes Rechteck in einer breiten Leiste, zwischen äußerer und innerer Leiste Taumuster. In der Mitte der gerundeten Platte ein lateinisches Kreuz mit sich verbreitenden Armen.

Datierung: -

Literatur: Tezcan, Topkap1, 45, Abb. 21.

V-8 Stück mit Lorbeerblattstab

Marmor

Ort: SM, K. 68/69, Stadtseite 
Maße konnte nicht aufgenommen werden

Beschreibung: Lorbeerblattstab von zwei flankierenden Leisten eingefaßt.

Datierung:-

Unpubliziert

V-9. Gesims (?) mit leerem Rahmen

Marmor

Ort: Mermerkule, Nordseite

Maße konnte nicht aufgenommen werden

Beschreibung: Eine Langseite sichtbar. Zwei schmucklose Rechteckrahmen mit Herzblättern an den Ecken der inneren Rahmen, dazwischen abgearbeiteter Knauf. Rechts abgearbeitet.

Datierung:-

Unpubliziert

V-10. Gesims (?) mit Akanthus

Marmor

Ort: SM, K. 9/10, Seeseite

Maße: Breite 78, Höhe $25 \mathrm{~cm}$

Beschreibung: Ragt aus dem Mauerverband heraus. Fußleiste nicht erhalten. Ein fünflappiges Akanthusblatt, die mittlere Rippe mit einem überfallenden Blatt. Schmucklose Kopfleiste. Stück ist links abgeschnitten, rechts neben den seitlichen Akanthuslappen unbearbeitet.

Datierung: 5./6. Jahrhundert

Literatur: Tezcan, Topkapı, Abb. 31. 